\title{
The frequency of bacterial contamination and diversity of drug resistance patterns in devices and staff of endoscopy and colonoscopy units
}

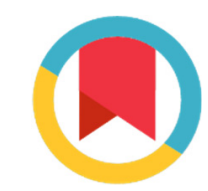

\author{
Parisa Torabi ${ }^{1}$, Masoumeh Azimirad ${ }^{1}$, Zahra Hasani ${ }^{1}$, Leili Afrisham², Masoud Alebouyeh ${ }^{1 *}$, Amir Houshang Mohammad \\ Alizadeh $^{3}$, Mohammad Reza Zali ${ }^{4}$
}

Received: 1 Aug 2016

Published: 18 Dec 2017

\begin{abstract}
Background: This study aimed at analyzing microbial contamination in medical equipment, environment, and staff of a gastroenterology unit.

Methods: Samples of gastrointestinal imaging devices, the environment, and staff were collected using standard swab-rinse technique and biochemical or molecular characteristics of the isolates, their susceptibility to antibiotics, and similarity of the resistance patterns were investigated.

Results: Out of 107 samples, bacterial contamination was detected in the hands of staff (54.1\%), imaging devices $(56.7 \%)$, and in the environment $(54.5 \%)$. While Pseudomonas spp. were detected only in the imaging devices (13.5\%), Bacillus spp. (32.4\% and $31.5 \%)$, Enterococcus spp. (14.3\% and 5.9\%), Clostridium difficile (10.8\% and 10.5\%), and Staphylococcus epidermidis (5.4\% and $15.9 \%$ ) were orderly the most common isolates from samples of the imaging devices and the environment. Nearly, $40 \%$ of $P$. aeruginosa strains were resistant to cefepime, while resistance to cephalosporins and $\beta$-lactamase inhibitor was detected in $33 \%$ and $75 \%$ of $S$. aureus strains, respectively. Homology of resistance patterns was detected between the imaging devices and hands of the staff.

Conclusion: Our results proposed biofilm and spore forming bacteria as main contaminants of imaging devices in this hospital. Homology of the resistance patterns proposed involvement of staff in contamination of the equipment.
\end{abstract}

Keywords: Imaging devices, Gastroenterology unit, Bacterial contamination, Antimicrobial Resistance, Cross-contamination

Copyright $₫$ Iran University of Medical Sciences

Cite this article as: Torabi P, Azimirad M, Hasani Z, Afrisham L, Alebouyeh M, Mohammad Alizadeh AH, Zali MR. The frequency of bacterial contamination and diversity of drug resistance patterns in devices and staff of endoscopy and colonoscopy units. Med J Islam Repub Iran. 2017 (18 Dec);31:104. https://doi.org/10.14196/mjiri.31.104

\section{Introduction}

Contamination of the medical devices is one of the widespread causes of hospital acquired infections (HAIs). Endoscopes and colonoscopes are the most widely used devices, whose role in development of HAIs is not well known. Several outbreaks of gastroenteritis in association with contaminated fiber optic imaging devices or biopsy forceps have been reported in different countries $(1,2)$.

Corresponding author: Dr Masoud Alebouyeh, masoud.alebouyeh@gmail.com

1. Foodborne and Waterborne Diseases Research Center, Research Institute for Gastroenterology and Liver Diseases, Shahid Beheshti University of Medical Sciences, Tehran, Iran.

2. Gastroenterology Unit of Ayatollah Taleghani Hospital, Shahid Beheshti University of Medical Sciences, Tehran, Iran.

3. Gastroenterology and Liver Diseases Research Center, Research Institute for Gastroenterology and Liver Diseases, Shahid Beheshti University of Medical Sciences, Tehran, Iran, \& Foodborne and Waterborne Diseases Research Center, Research Institute for Gastroenterology and Liver Diseases, Shahid Beheshti University of Medical Sciences, Tehran, Iran.

4. Gastroenterology and Liver Diseases Research Center, Research Institute for Gastroenterology and Liver Diseases, Shahid Beheshti University of Medical Sciences, Tehran, Iran, \& Basic and Molecular Epidemiology of Gastrointestinal Disorders Research Center, Research Institute for Gastroenterology and Liver Diseases, Shahid Beheshti University of Medical Sciences, Tehran, Iran.
These infections usually occur after examination of patients with inadequately disinfected medical devices. Contamination of endoscopes and colonoscopes can occur through direct contact of patients with the medical tools that are infected with body secretion or through indirect contact of employees and personnel with patients and infected equipment. Ability of some bacteria to form bio-

$\uparrow$ What is “already known" in this topic:

Inappropriate washing and sterilization of medical devices are considered as main causes of hospital acquired infection during endoscopy and colonoscopy.

\section{$\rightarrow$ What this article adds:}

Our results proposed biofilm and spore forming bacteria as main contaminants of imaging devices in the studied hospital. Resistance of these strains to broad spectrum antibiotics and homology of the resistance patterns with those isolated from staff proposed designation of permanent hygienic programs in each hospital. 
films on the inner side of the devices and the related equipment can contribute to failure of the decontamination process. In the present study, bacterial contamination of imaging devices of a gastroenterology unit and the personnel in their contact was examined to investigate the rate of cross- contamination among them.

\section{Methods}

\section{Sampling and bacterial identification}

Random specimens were prepared from endoscope, colonoscope, ultrasonography endoscope, forceps, patient's bed, and plate of cleansing imaging devices before examination and immediately after the sterilization process during February 2010 and September 2010. The specimens were also collected from the personnel of the endoscopy unit (including hands, nose, and cell phones) at the time of examination. Accordingly, the sterilized swabs saturated with physiological serum $(\mathrm{pH}=7)$ were cultured on Blood agar and MacConkey agar media and incubated in aerobe condition at $37^{\circ} \mathrm{C}$ for 24 hours. The biochemical characteristics of all isolates were verified using Bergey's Manual of Systematic Bacteriology. Clostridium difficile was identified in selective culture medium supplemented with $7 \%$ horse blood and selective components and PCR (3).

\section{Antimicrobial susceptibility testing}

To analyze the susceptibility of our isolates to antibiotics, different commercially antibiotic discs (Padtan Teb, Iran) were used for each bacterial genus. In case of Bacillus cereus, interpretation of results was performed using the criteria described for $S$. aureus. Multiple drug resistance (MDR) was defined in those isolates that were resistant to at least 3 or more antibiotics from different categories (4). Methicillin resistant S. aureus (MRSA) phenotype was determined based on resistance of $S$. aureus strains to cefoxitin $(30 \mu \mathrm{g} / \mathrm{mL})$ in Mueller Hinton agar medium. Pseudomonas aeruginosa ATCC 27853 and S. aureus ATCC 25923 strains were used as control strains.

\section{Determination of the phenetic similarity}

To analyze probable and basic similarities between the strains obtained from the personnel and medical equipment, drug resistance patterns were applied as biotyping numerical data. Accordingly, taxonomic similarity of the resistance patterns was assessed using NTSYSpc software.

\section{Results}

\section{Sample collection and bacterial contamination}

Out of 107 samples, bacterial contamination was observed in hands of the staff of gastroenterology unit with a frequency of $54.1 \%(26 / 48)$ and in the imaging devices with a frequency of $56.7 \%$. Main bacterial isolates from the imaging gastroenterology devices and the environmental samples were orderly included $S$. aureus, Enterococcus spp., S. epidermaidis, Bacillus spp., Pseudomonas spp. and C. difficile. S. aureus, S. epidermidis, Enterococcus spp. and Bacillus spp were detected in hands of health care staff in the gastroenterology unit. Coexistence of different bacterial genera in a single sample was detected among $8.1 \%(3 / 37)$ and $13.6 \%(3 / 22)$ of the imaging devices and environmental samples, respectively. Types of these mixed infections and their frequencies are depicted in Table 1.

\section{Antibiotic susceptibility testing}

According to the antibiotic susceptibility test results, 3

Table 1. Frequency of bacterial contamination in gastroenterology unit of a hospital in Tehran, Iran

\begin{tabular}{|c|c|c|c|c|c|c|c|c|c|}
\hline 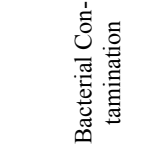 & 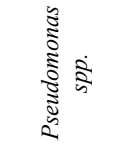 & 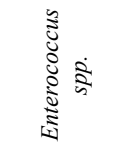 & $\dot{\omega}$ & 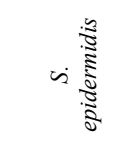 & 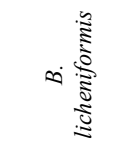 & $\infty \frac{\tilde{z}}{\frac{\tilde{z}}{\tilde{g}}}$ & 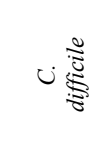 & vi: & $\begin{array}{l}\text { Mixed } \\
\text { infection }\end{array}$ \\
\hline $\begin{array}{l}\text { Colonoscope } \\
(7 / 14,50 \%)\end{array}$ & $1(7.1 \%)$ & $2(14.3 \%)$ & - & $1(7.1 \%)$ & $4(28.6 \%)$ & - & $\begin{array}{c}2 \\
(14.3 \%)\end{array}$ & - & $\begin{array}{c}2 / 14(14.3 \%) \\
\text { S.epidermidis }+ \text { B. licheniformis } \\
\text { or } \\
\text { P. aeruginosa + Enterococcus + B. } \\
\text { licheniformis }\end{array}$ \\
\hline $\begin{array}{l}\text { Endosono- } \\
\text { graphic device } \\
(3 / 5,60 \%)\end{array}$ & - & - & - & - & $1(20 \%)$ & $1(20 \%)$ & $1(20 \%)$ & - & 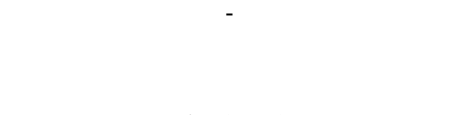 \\
\hline $\begin{array}{l}\text { Endoscope } \\
(11 / 18,61.1 \%)\end{array}$ & $4(22.2 \%)$ & - & - & $1(5.5 \%)$ & $5(27.7 \%)$ & $1(5.5 \%)$ & $1(5.5 \%)$ & - & $\begin{array}{c}1 / 18(5.5 \%) \\
\text { P. aeruginosa + C. difficile }\end{array}$ \\
\hline $\begin{array}{l}\text { Hands of } \\
\text { HCWs a } \\
(26 / 48,54.1 \%)\end{array}$ & - & $1(2 \%)$ & $8(16.6 \%)$ & $16(33.3 \%)$ & $8(16.6 \%)$ & $1(2 \%)$ & - & - & $\begin{array}{c}5 / 48(10.4 \%) \\
\text { S. epidermidis + B. licheniformis; S. aureus + } \\
\text { B. coagulans; } \\
\text { S. aureus + B. cereus; } \\
\text { S. aureus + S. epidermidis } \\
\text { or S. aureus and B. licheniformis }\end{array}$ \\
\hline $\begin{array}{l}\text { Washing } \\
\text { Equipment }\end{array}$ & - & - & - & $1(50 \%)$ & - & $1(50 \%)$ & - & - & $\begin{array}{c}\text { 1/2 (50\%), S. epidermidis }+ \\
\text { B. coagulans- }\end{array}$ \\
\hline $\begin{array}{l}\text { Forceps } \\
(1 / 2,50 \%)\end{array}$ & - & - & - & - & - & - & $1(50 \%)$ & - & \\
\hline $\begin{array}{l}\text { Patients'bed } \\
(10 / 17,58.8 \%)\end{array}$ & - & $1(5.9 \%)$ & $2(11.7 \%)$ & $2(11.7 \%)$ & $5(29.4 \%)$ & - & $1(5.9 \%)$ & $1(5.9 \%)$ & $\begin{array}{c}2 / 17(11.7 \%) \\
\text { S. aureus }+ \text { B. licheniformis } \\
\text { or } \\
\text { B. licheniformis }+ \text { C. difficile }\end{array}$ \\
\hline
\end{tabular}

a. HCWs: Health care workers. 
out of $12 S$. aureus strains were defined as MRSA (25\%). MDR $S$. aureus was detected among $75 \%$ of the strains (9/12), which was included in all the 3 MRSA strains. The highest rate of resistance among the studied bacterial genera was observed against penicillin $(S$. aureus, 100\%; S. epidermidis, 87.5\%; Enterococcus spp., 75\%; and B. cereus, $100 \%$ ). Resistance to ampicillin, vancomycin, gentamicin, and nitrofurantoin was detected among 75\% (2/3) of Enterococcus strains. P. aeruginosa strains were sensitive to most of the antibiotics tested, but resistant to cefepime $40 \%(2 / 5)$. Resistance to third and fourth generation cephalosporins was detected in strains of $S$. aureus (4/12, 33\%), B. cereus $(1 / 1,100 \%)$, and S. epidermidis $(6 / 24,25 \%)$. All the bacteria were sensitive to imipenem, and resistance to ciprofloxacin was detected just for Enterococci $(2 / 3,75 \%)$. Resistance to $\beta$-lactamase inhibitor was mainly observed among the strains of $S$. aureus $(11 / 12,91.6 \%)$ and $S$. epidermidis $(10 / 24,41.6 \%)$. The sole B. cereus strain was sensitive to ciprofloxacin, vancomycin, gentamicin, imipenem, and chloramphenicol. Moreover, triple, quadruple, and senary drug resistance phenotypes among $S$. aureus and $S$. epidermidis strains were orderly detected in a frequency of $44.4 \%$ (4/9) and $59.1 \%(13 / 22), 44.4 \%(4 / 9)$ and $13.63 \%(3 / 22)$, and $11.1 \%(1 / 9)$ and $4.54 \%(1 / 22)$. Quintuple drug resistance phenotype was detected just for $S$. epidermidis strains $(18.18 \%(4 / 22)$.

\section{Analysis of the phenetic similarity}

Homology results revealed the highest similarity among S. epidermaidis strains, while no homology was found for $S$. aureus strains among different samples. These similarities were observed between the personnel's hands, or between the personnel's hand and patients' table (Fig. 1). This dendrogram was drawn using NTSys software, Unweighted Paired Group Method, with Arithmetic mean (UPGMA) algorithm after conversion of the resistance data to 0 (sensitive) and 1 (resistance) numeric matrix. The abbreviation letters are related to sampling parts: $\mathrm{H}$ : hand, W: washing system, C: colonoscope, B: patient's bed, M: mobile, T: patient's table, and E: endoscope. The numbers below the diagram presented the percentage of homology.

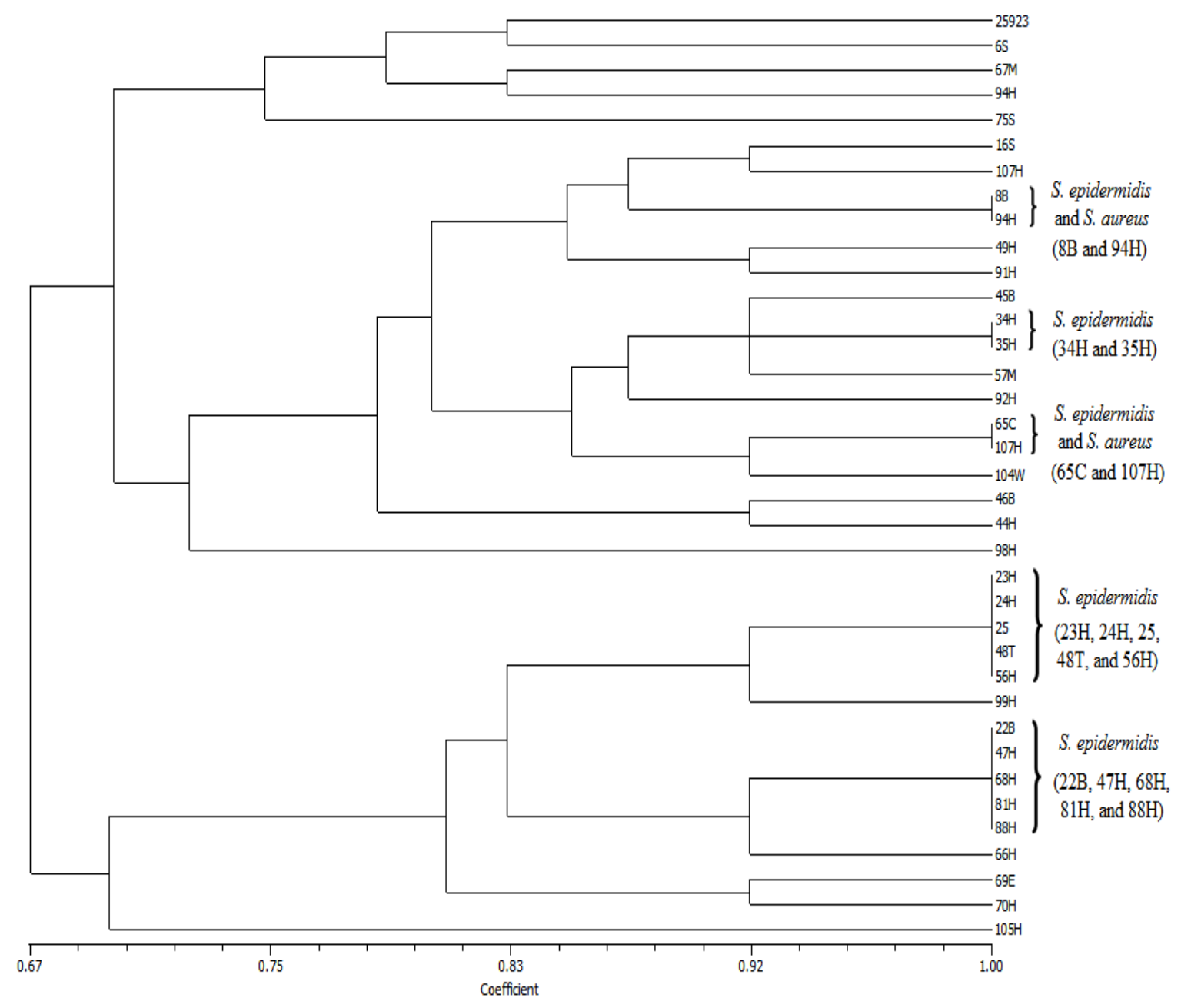

Fig.1. Phenotypic homology dendrogram of drug resistance patterns among S. aureus and S. epidermaidis strains isolated from various sources in gastroenterology unit 


\section{Discussion}

The presence of pathogenic bacteria, such as Enterococcus, Pseudomonas and $C$. difficile species, in the samples collected from the endoscope and colonoscope confirmed the weakness of the used sterilization methods for these medical imaging devices. We found an association between the cleaning conditions and types of the characterized bacteria in these devices, as most of these bacteria are generally resistant to the administrated cleaning solution (Glutaraldehyde 2\% for 10 minutes). Previous studies have revealed that bacterial spores and capsule forming bacteria can sustain their life due to their innate resistance to these compounds and can transfer easily between patients and hospital environment $(5,6)$. S. aureus, S. epidermaidis, S. Saprophyticus, and Enterococcus strains among the Gram- positive bacteria and $P$. aeruginosa strains among the Gram- negative bacteria are known as the main bacteria capable of biofilm formation in the medical devices. Bacillus spp. and Clostridia are spore forming bacteria, whose elimination needs disinfection through glutaraldehyde (2\%-3.4\%) for 20 minutes (7).

Bacillus spp. and Pseudomonas spp. were among the commonest bacterial species detected in the studied equipment. In a study in the USA and Canada, Gramnegative bacteria including Salmonella, E. coli, Pseudomonas, Klebsiella, Enterobacter species, Serratia marcescens, and Helicobacter pylori were introduced as the most common transmitting bacteria by endoscopes and colonoscopies through imperfective sterilization of the equipment and use of infected water sources in rinse (1). High frequency of Bacillus species and $C$. difficile in medical imaging equipment in the present study could be attributed to the resistance of their spores to the used sterilization process. Colonization of $P$. aeruginosa in these devices, which is known as a biofilm forming bacteria, supports this hypothesis.

In a study by McFarland LV. et al., the existence of $C$. difficile was reported in 59\% of HCWs' hands. Although transmission of $C$. difficile by HCWs was not confirmed in our study, occurrence of cross- contamination between patients and hospital environment was suggested because of its isolation from the hospital environment and accessory equipment of the imaging devices.

Results of antimicrobial susceptibility testing revealed a high frequency of MDR $S$. aureus and $S$. epidermidis in the gastroenterology imaging unit. Most of these strains were isolated from HCWs hands and patients' bed. Resistance of these strains to broad spectrum $\beta$-lactams and $\beta$-lactam inhibitors has made them hospital adapted strains. Homology of the resistance patterns between the Staphylococcus isolates from imaging devices and hands of HCWs proposed the occurrence of cross- contamination in this hospital. Such relationship was suggested before by Alighardashi M. et al. and Sedighi I. et al $(8,9)$.

MRSA can cause antibiotic associated diarrhea in hospitalized patients. Contamination of colonoscope during handling of contaminated staff will increase the risk of their transmission to these patients. Environment including washing equipment is another possible source of infection in the gastroenterology unit. In our study, we de- tected contamination of washing equipment with spore forming bacteria, B. coagulans, and S. epidermidis. Detection of same bacteria in the hands of the staff proposed their involvement in contamination of the equipment during the washing procedure and handling. However, such correlation was not determined for $C$. difficile, but its presence in both patents' bed and the equipment proposed an increased risk of contamination of the patients by the environment or equipment in this unit.

\section{Conclusion}

Our results proposed biofilm and spore forming bacteria as the main contaminant of imaging devices in this hospital. Homology of the resistance patterns proposed involvement of staff in contamination of the equipment. Designation of hygienic programs should be considered to prevent HAIs through contaminated imaging devices in hospital settings.

\section{Acknowledgements}

This study was kindly supported by a research grant (Code: 613) from Foodborne and Waterborne Diseases Research Center, Research Institute for Gastroenterology and Liver Diseases, Shahid Beheshti University of Medical Sciences, Tehran, Iran. Authors of this article would like to sincerely thank all colleagues for cooperating in conducting this research.

Conflict of Interests

The authors declare that they have no competing interests.

\section{References}

1. Dwyer DM, Klein EG, Istr GR, Robinson MG, Neumann DA, McCoy GA. Salmonella newport infections transmitted by fiberoptic colonoscopy. Gastrointest Endosc. 1987;33(2):84-87.

2. Epstein L, Hunter JC, Arwady MA, Tsai V, Stein L, Gribogiannis M, et al. New Delhi metallo- $\beta$-lactamase-producing carbapenem-resistant Escherichia coli associated with exposure to duodenoscopes. JAMA. 2014;312(14):1447-1455.

3. Parhizkar B, Alebouyeh M, Dezfulian A, Azimi Rad M, Bahreini B, Nazemalhosseini E, et al. Coexistence of enterotoxigenic Staphylococcus aureus and cytotoxic Clostridium difficile as predisposing factors for septic shock in patients with inflammatory bowel disease. Prz Gastroenterol. 2013;8(3):206-210.

4. Magiorakos AP, Srinivasan A, Carey RB, Carmeli Y, Falagas ME, Giske CG, et al. Multidrug-resistant, extensively drug-resistant and pandrug-resistantbacteria: an international expert proposal for interim standard definitions for acquired resistance. Clin Microbiol Infect. 2012;18(3):268-281.

5. Gerding DN, Muto CA, Owens RC. Measures to control and prevent Clostridium difficile infection. Clin infect dis. 2008;46(1):S43-9.

6. Azimirad M, Krutova M, Nyc O, Hasani Z, Afrisham L, Alebouyeh M, Zali MR. Molecular typing of Clostridium difficile isolates cultured from patient stool samples and gastroenterological medical devices in a single Iranian hospital. Anaerobe. 2017;47:125-128.

7. Kovaleva J, Peters FTM, van der Mei HC, Degener JE. Transmission of Infection by Flexible Gastrointestinal Endoscopy and Bronchoscopy. Clin Microbiol Rev. 2013;26(2):231-254.

8. Alighardashi M, Aeini M, Naeinian F, Mohamadi H. The Amount and Type of Microbial Contamination on Cell Phones of Medical Staff in Shahid Beheshti Hospital, Hamadan, Iran. J Health System Research. 2012;7(6):1-9.

9. Sedighi I.; Alikhani MY; Ramezani S; Nazari M; Mozaffari Nejad AS Bacterial Contamination of Mobile Phones of Health Care Providers in a Teaching Hospital in Hamadan Province, Iran. Arch Clin Infect Dis. 2015; 10(2): e22104. 